\title{
Joint use of circuit models and complexor diagrams in generator design
}

\author{
Ö. Göl ${ }^{1}$ and B. Sobhi-Najafabadi ${ }^{2}$ \\ ${ }^{1}$ School of Electrical and Information Engineering \\ University of South Australia \\ GPO Box 2471, Adelaide 5001, Australia \\ Telephone: +61883023285 \\ Facsimile: +6183023384 \\ E-mail: Ozdemir.Gol@unisa.edu.au \\ ${ }^{2}$ School of Advanced Manufacturing and Mechanical Engineering \\ Centre for Advanced Manufacturing Research \\ University of South Australia \\ GPO Box 2471, Adelaide 5001, Australia \\ Telephone: +61883022018 \\ Facsimile: +6183025292 \\ E-mail: Bijan.Sobhi-Najafabadi@unisa.edu.au
}

\begin{abstract}
Impedance models have been routinely used in generator design. Despite their many limitations, they are indispensable for rapid performance evaluation, which makes them also suitable for use in conjunction with design optimisation algorithms.

This paper discusses the combined use of impedance models and complexor diagrams in a.c. generator design. The approach is demonstrated by applying it to the design of a permanent magnet synchronous generator with surface mounted magnets on the rotor.
\end{abstract}

\section{Key words}

Circuit models, complexor diagrams, generator design, performance prediction, permanent magnet generator

\section{Introduction}

Lumped parameter circuit models have been widely used in the performance analysis and design of electrical machines. They are capable of predicting the overall machine performance with reasonable accuracy under steady state operating conditions. In the case of a synchronous generator they look deceptively simple, yet their use is crucial, especially to the initial dimensioning of the machine at the design stage. As such, they are indispensable for rapid performance evaluation, which makes them suitable for use in conjunction with design optimisation algorithms.
This paper deals with the joint use of impedance models and complexor diagrams in a.c. generator design to enhance the effectiveness of the design effort, demonstrating its usefulness by applying it to the design of a permanent magnet synchronous generator with surface mounted magnets on the rotor.

\section{Circuit Models}

Circuit models have traditionally been the primary tools in the design of electrical machines. They are formed either as electrical circuit models or magnetic circuit models, representing the electromagnetic features of the machine. When used together with other modelling aids, such as complexor diagrams, they do provide considerable insights into the causeeffect relationships of machine preformance. In spite of their apparent simplicity, model parameters are still rather complex functions of machine geometry and material properties and must be obtained with utmost care.

Fig. 1 depicts a permanent magnet generator with a cylindrical rotor, with surface mounted magnets on the rotor.

The low order steady state impedance model for the generator, assuming core linearity, is given in Fig. 2.

\section{Complexor Diagrams}

Complexor diagrams combine time and space phasors, representing magnitude and space relationships between electrical and magnetic quantities $[1,2,3]$. 


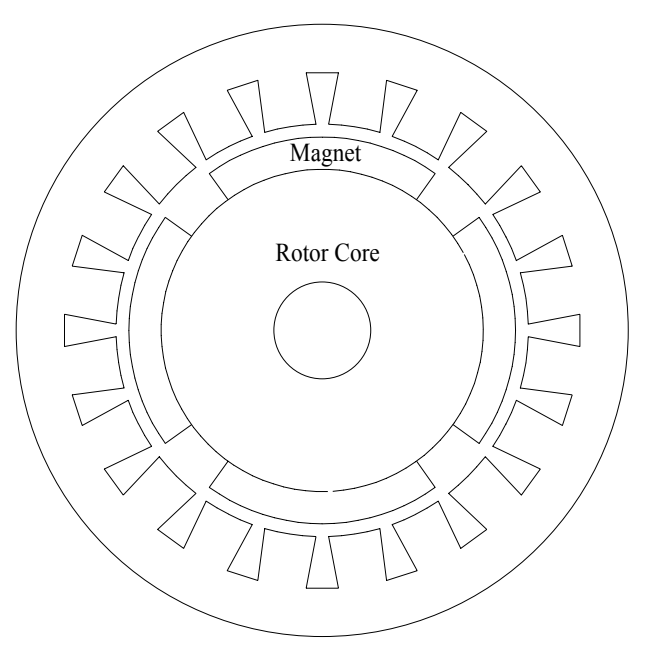

Fig. 1. Permanent magnet synchronous generator with surface mounted magnets on the rotor

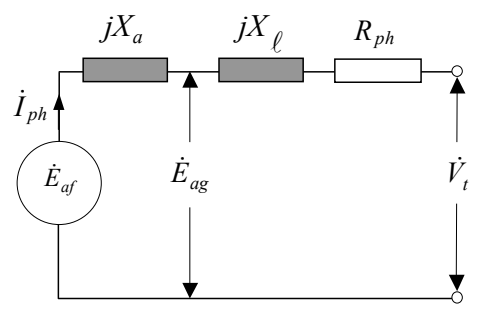

Fig.2. Low order steady state impedance model with linear parameters

Using complexor diagrams, one can derive information on the internal magnetic and electrical quantities on the basis of terminal characteristics. The reverse also holds: when the MMFs and their spatial alignments within the machine are known, then terminal quantities can be determined.

Fig. 3 depicts the complexor diagram for a generator with a cylindrical rotor and surface mounted magnets on the rotor. In this case it is assumed that the core is not subject to saturation, leading to the model depicted in Fig. 2.

The terminal voltage equation for the generator with linear core can then be formulated as

$\dot{V}_{t}=\dot{E}_{a g}-\dot{I}_{p h} R_{p h}-j \dot{I}_{p h} X_{\ell}$

or alternatively as:

$\dot{V}_{t}=\dot{E}_{a f}-\dot{I}_{p h} R_{p h}-j \dot{I}_{p h}\left(X_{\ell}+X_{a}\right)$

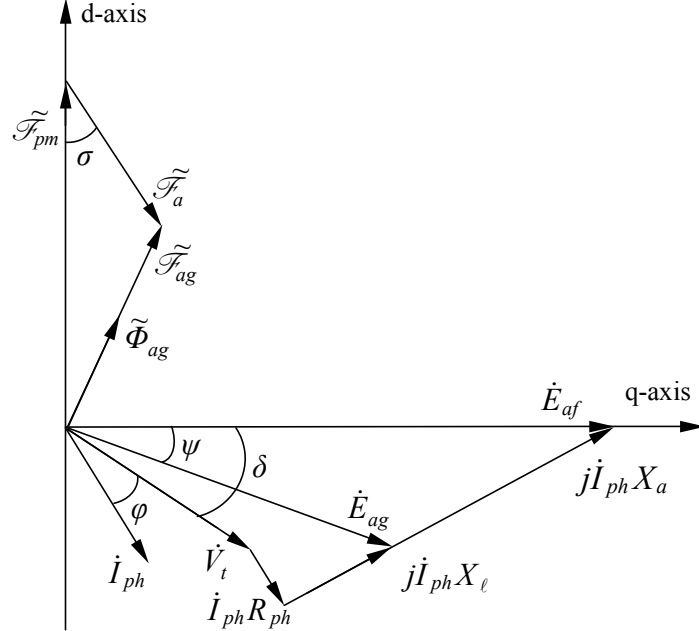

Fig. 3. Complexor diagram for a permanent magnet machine with surface mounted magnets (no core saturation)

If saturation (that is, core non-linearity) is taken into account, this must be reflected in the model as depicted in Fig. 4. Saturation effects can be accounted for by modifying both the impedance model and the associated complexor diagram. Fig.s 4 and 5 illustrate these modifications by the inclusion of non-linear elements.

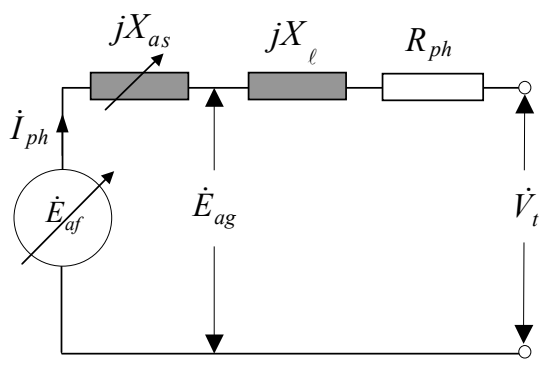

Fig. 4. Per phase impedance model of a permanent magnet generator accounting for non-linearities

For a permanent magnet generator with a cylindrical rotor, the air gap mmf, $\tilde{\mathscr{F}}_{a g}$, representing the combined effect of permanent magnet excitation $\widetilde{\mathscr{F}}_{p m}$ and armature reaction, can be expressed as

$\tilde{\mathscr{F}}_{a g}=\widetilde{\mathscr{F}}_{p m}+\widetilde{\mathscr{F}}_{a}$

The magnitude of $\tilde{\mathscr{F}}_{a g}$ is given as

$\left|\tilde{\mathscr{F}}_{a g}\right|=\sqrt{\left|\tilde{\mathscr{F}}_{p m}\right|^{2}+\left|\tilde{\mathscr{F}}_{a}\right|^{2}-2\left|\tilde{\mathscr{F}}_{p m}\right|\left|\tilde{\mathscr{F}}_{a}\right| \cos \sigma}$ 


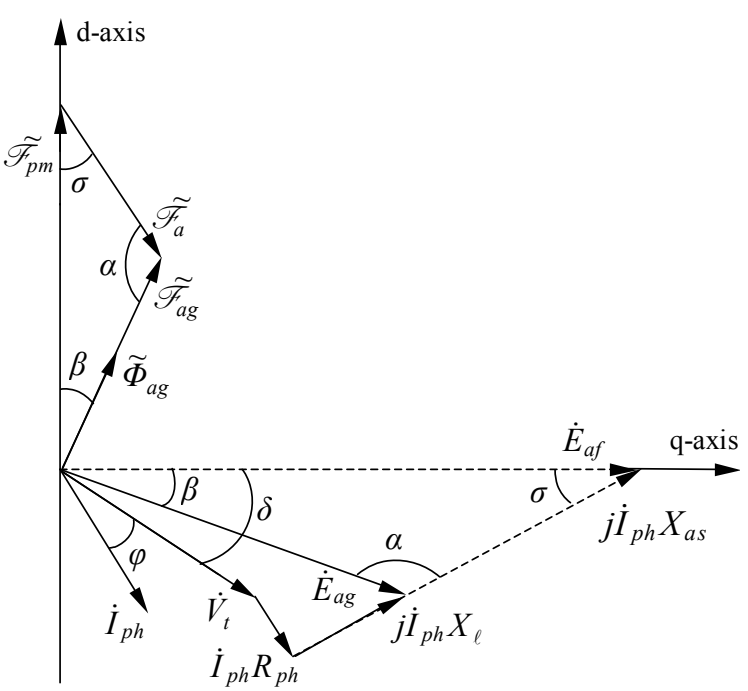

Fig. 5. Non-linear complexor diagram of permanent magnet generator

Knowledge of $\widetilde{\mathscr{F}}_{a g}$ enables $\dot{E}_{a g}$ to be calculated. It must be noted that if saturation is to be accounted for $\dot{E}_{a g}$ can no longer be obtained from a simple superposition of the constituent components, but can only be determined on the basis of iterative computations. $X_{a s}$ in Fig. 5 denotes the saturated armature-reaction reactance. This reactance is smaller than its linear counterpart when the machine is working in saturation and varies with load.

\section{The Design Procedure}

Designing a generator involves resolving the following requirements:

- Design specification

- Core design

- Winding design

- Performance prediction

- Optimisation

Evidently design specification comprises user requirements and evokes issues of choice of topology and materials. The next most important challenge is the core design: geometrical data such as yoke and teeth dimensions, number of poles, number of teeth, internal and external diameters must be determined taking into account the properties of the materials adopted for use. The overall dimensioning is aided by the relationship:

$S=\left(1.11 \pi^{2} K_{w} B_{a v} Q_{s e l}\right) D_{b}^{2} L_{c} n$ where

$S$ generated apparent power, VA

$K_{w}$ winding factor

$Q_{\text {sel }}$ specific electrical loading, $\mathrm{A} / \mathrm{m}$

$B_{a v}$ specific magnetic loading, $\mathrm{Wb} / \mathrm{m}^{2}$

$D_{b}$ stator bore diameter, $\mathrm{m}^{2}$

$L_{c} \quad$ stator core length, $\mathrm{m}$

$n$ rotor synchronous speed, $1 / \mathrm{s}$

Equation (5) is significant in that it helps determine the base dimensions like the stator bore diameter $\left(D_{b}\right)$ and the core length $\left(L_{c}\right)$. Core details such as the number and shape of slots are determined next on the basis of magnetic, electrical and mechanical considerations $[4,5,6,7]$. Then comes the design of the armature windings. Knowledge of winding details enables the magnitude of the air gap flux required for generating the EMF, and hence the terminal voltage to be determined. The subsequent rotor core design, of which magnets constitute an integral part, ensures the provision of the air gap flux needed to achieve the specific requirements. Evidently, this entails working out details such as the air gap clearance, magnet volume and topology, and rotor core geometry. The validity of the design in meeting the specification must be checked next. This is done with the aid of suitable design models, including complexor diagrams.

Determining the details of core geometry for an electrical machine toward satisfying the requirements of a given design specification constitutes a challenge. The task is complex because of the numerous choices available in terms of design parameters such as dimensions, shape and slot numbers. The task is also onerous, since the wrong choices can perilously affect the performance, ultimately rendering the machine useless. This may explain the reason as to why many manufacturers prefer to utilise existing lamination designs since they feel safe on account of these already being "tested" before, even though such may leave much to be desired when it comes to optimality.

Winding design has been viewed as part art and part science by some [8]. Determination of the intricate details of a winding to be housed in a given core is by no means a trivial task. Even though the number of phases may be fixed by the design specification, there are many variables, which need to be determined. These include number of turns per phase as well as the actual type of winding. 
Nevertheless, once the lamination topology is known, winding design is reasonably straightforward. Equipped with the knowledge of the phase current, $I_{p h}$, calculated as

$I_{p h}=\frac{P}{m V_{p h} \cos \varphi}$

where $\mathrm{m}, V_{p h}$, and $\cos \varphi$ are number of phases, phase voltage and power factor respectively, and ensuring that the current density does not exceed $5 \times 10^{6} \mathrm{~A} / \mathrm{m}^{2}$, a wire gauge can be selected from the available standard wire gauges. Slot geometry determines the maximum number of conductors, which can be fitted into the slot space. The slot space accommodating active conductors is in the order of $60-70 \%$ of the actual slot space due to a number of factors including wire insulation and slot wedges.

For a double-layer, integral-slot lap-winding (chorded by one slot) the number of conductors per slot, $N_{c s}$, should be even. Thus, the number of turns per phase can be found as

$N_{p h}=\frac{N_{s s} N_{c s}}{2 m}$

$N_{s s}$ is the number of stator slots.

Now, one can proceed to determine the stator core length. This can be done considering the relationship between the generated phase $\mathrm{EMF}, E_{p h}$, and the corresponding air gap flux per pole, $\Phi$, required at full-load. The generated phase EMF is commonly known as the "air gap EMF". To reflect this, the symbol $E_{a g}$ will be used to substitute for $E_{p h}$ from now on. In terms of the base dimensions and air gap flux $E_{a g}$ is given by

$E_{a g}=\frac{4.44 K_{w} N_{p h} f_{s} B_{a v} \pi D_{b} L_{c}}{p}$

where

$p \quad$ number of poles

$N_{p h}$ number of turns per phase

$f_{s}$ synchronous frequency, $\mathrm{Hz}$

$E_{a g}$ can also be expressed, by referring to the phasor components of the complexor diagram in Fig. 5 (reproduced in Fig. 6 for clarity) as

$$
\left|\dot{E}_{a g}\right|=\sqrt{\left(\left|\dot{V}_{t}\right| \cos \varphi+\left|\dot{I}_{p h} R_{p h}\right|\right)^{2}+\left(\left|\dot{V}_{t}\right| \sin \varphi+\left|\dot{I}_{p h} X_{\ell}\right|\right)^{2}}
$$

Substituting $V_{p h}$ for $\left|\dot{V}_{t}\right|$ and $I_{p h}$ for $\left|\dot{I}_{p h}\right|$ into Equation (9), one obtains

$$
\left|\dot{E}_{a g}\right|=\sqrt{\left(V_{p h} \cos \varphi+I_{p h} R_{p h}\right)^{2}+\left(V_{p h} \sin \varphi+I_{p h} X_{\ell}\right)^{2}}
$$

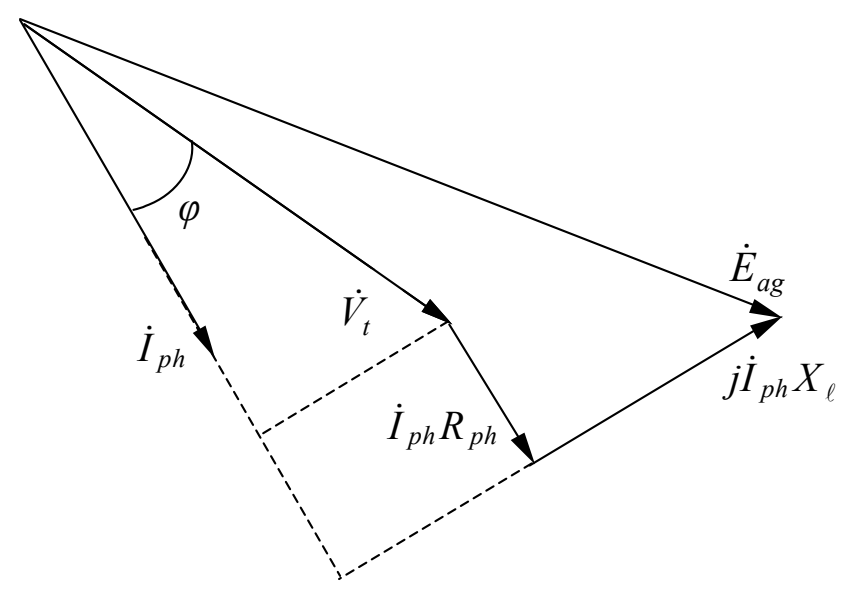

Fig. 6. Phasor diagram for generated air gap emf

In Equation (10), both $R_{p h}$ and $X_{\ell}$ are functions of $L_{c}$. In Equation (8) all the right hand terms are known except $L_{c}$. Thus, $L_{c}$ and $E_{a g}$ can be calculated from Equations (8) and (10) by iteration. In each iteration, starting with a small value of $L_{c}$, EMF values from the two equations are compared till the difference diminishes. Then, the required air gap flux per pole at full-load $\Phi$ can be determined as

$\Phi=\frac{B_{a v} \pi D_{b} L_{c}}{p}$

\section{Performance Prediction}

In analysing the behaviour of a saturated permanent magnet synchronous generator by means of complexor diagrams, it is prudent to rely on the wellestablished procedures of synchronous generator analysis $[1,6,9]$. In the case of a permanent magnet generator with surface mounted magnets, the method of analysis for conventional synchronous generator with a cylindrical rotor can be adopted for performance analysis. It should be noted that 
performance analysis is tantamount to predicting the terminal voltage for a given load condition, which can be determined with the aid of Fig. 5 .

In the MMF triangle of Fig. 5, the magnet MMF $\widetilde{\mathscr{F}_{p m}}$ is known both in magnitude and alignment. $\widetilde{\mathscr{F}}_{a}$ is known in magnitude, but its direction must be determined by successive iterations. Evidently, $\widetilde{\mathscr{F}}_{a g}$ must be obtained before $\widetilde{\Phi}_{a g}$, and hence $\dot{E}_{a g}$ can be determined. The difficulty arises from the fact that in a permanent magnet generator the excitation field is not controllable; terminal voltage is a function of the load current and power factor. This is in contrast with a conventional synchronous generator, where the excitation field is controllable, by means of which the specified terminal voltage for a given load can be attained.

Thus, one has to start with the mmf triangle in Fig. 5 by assuming an initial value for the angle $\sigma$ with which the magnitude of $\widetilde{\mathscr{F}}_{a g}$ can be determined tentatively as

$\left|\tilde{\mathscr{F}}_{a g}\right|=\sqrt{\left|\tilde{\mathscr{F}}_{p m}\right|^{2}+\left|\tilde{\mathscr{F}}_{a}\right|^{2}-2\left|\tilde{\mathscr{F}}_{p m}\right|\left|\tilde{\mathscr{F}}_{a}\right| \cos \sigma}$

The knowledge of $\widetilde{\mathscr{F}}_{a g}$ allows the flux $\widetilde{\Phi}_{a g}$ to be found by means of the MMF triangle in Fig. 5, representing the saturated case [9]. This leads to the calculation of $\left|\dot{E}_{a g}\right|$ as

$\left|\dot{E}_{a g}\right|=4.44 K_{w} f N_{p h}\left|\widetilde{\Phi}_{a g}\right|$

Once $\dot{E}_{a g}$ is known, $\dot{V}_{t}$ can be obtained by accounting for the voltage drops in stator phase resistance and leakage reactance. If the phase angle of $\dot{V}_{t}$ with the current $\dot{I}_{p h}$ does not satisfy the required power factor, the steps must be repeated, by choosing a different $\sigma$, until it does.

Some observation is in order in Fig. 5 concerning the EMF $\dot{E}_{a f}$. This component of the phasor diagram can no longer be found by simple superposition as in the linear case. It can only be determined after $\dot{V}_{t}$ has been calculated as described above. Extending the reactive voltage drop phasor to intersect with the quadrature axis yields its magnitude. The value of the saturated armature reaction reactance can then be calculated from $j \dot{I}_{p h} X_{a s}$ for each load condition. The value of $X_{a s}$ decreases as the machine is driven into saturation.
Performance characteristics of the generator can be obtained point-by-point by the process described above. The resolution of the characteristic, however, is dependent on the step size of the load current selected. The finer the step size, the longer it takes to produce the characteristic, especially when the machine is driven into saturation, since the iterative computations necessary are extensive and timeconsuming.

Fig. 7 shows the predicted external characteristic depicting the terminal behaviour of the generator [6, 9] under varying load conditions. Measured points are superimposed for comparison. Fig. 8 gives the efficiency as a function of load as predicted as well as measured. In both cases the comparison is favourable, substantiating confidence in the validity of the design approach adopted.

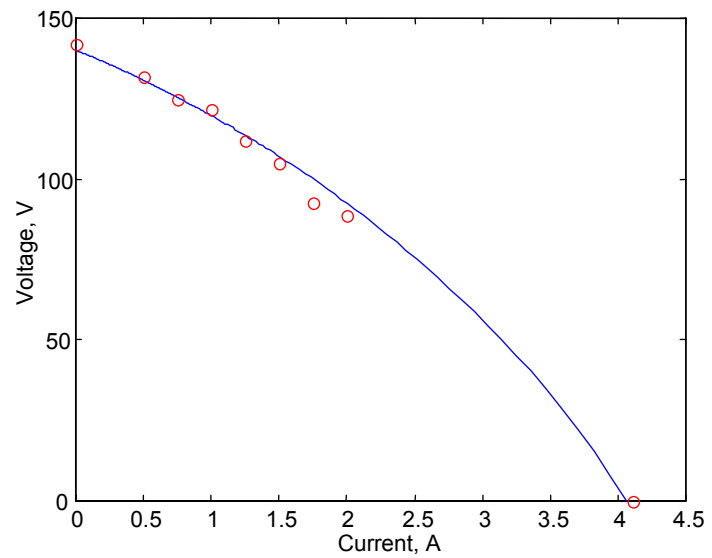

Fig. 7. External characteristic for unity power factor - Predicted 0 ○ Measured

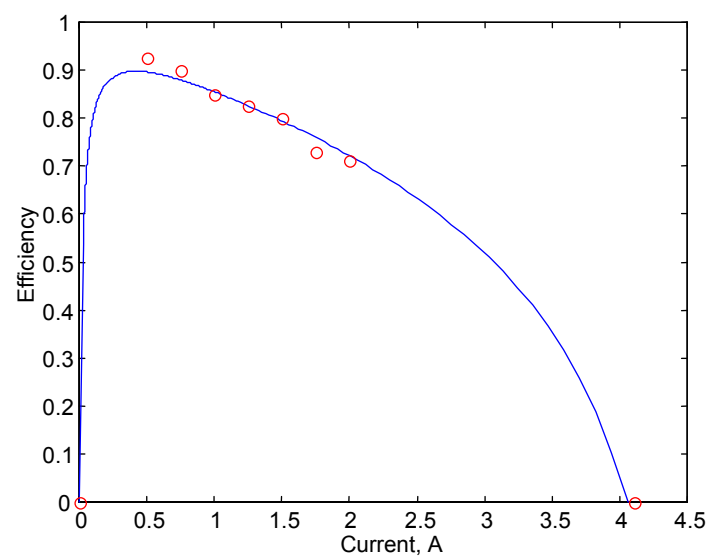

Fig. 8. Efficiency versus load current for unity power factor

— Predicted $\bigcirc \circ$ Measured 


\section{Conclusion}

Synchronous generator design is a challenge in itself. However design of a permanent magnet generator can be a greater challenge still. This is mainly due to the lack of well established design procedures for general use. The joint use of circuit models and complexor diagrams provides a practical means of designing a permanent magnet generator with a satisfactory level of confidence.

\section{References}

[1] J. Hindmarsh, Electrical Machines and Their Applications, Fourth Edition, Pergamon Press, Oxford, U.K., 1984.

[2] N. A. O. Demerdash, Computerized Magnetic Field Model for Performance Calculation of TurboGenerators with Asymmetrical and Symmetrical Rotors, PhD Thesis, University of Pittsburgh, U.S.A., 1971.
[3] M. Liwschitz-Garik and C. C. Whipple, AlternatingCurrent Machines, Second Edition, D. Van Nostrand Company, U.S.A., 1961.

[4] A. K. Sawhney, A Course in Electrical Machine Design, Fourth Edition, Dhanpat Rai \& Sons, Delhi, India, 1977.

[5] A. Still, and C. S. Siskind, Elements of Electrical Machine Design, Third Edition, McGraw-Hill Book Company, Inc, New York, U.S.A., 1954.

[6] Ö. Göl and B. Sobhi-Najafabadi, "Permanent Magnet Generator Design: A Systematic Approach", Proc. International Aegean Conference on Electrical Machines and Power Electronics, ACEMP 2001, Kuşadas1, Turkey, 2001, pp. 361-365.

[7] B. Sobhi-Najafabadi, An Enhanced Methodology for Permanent Magnet Generator Design, MEng (Research) Thesis, University of South Australia, Adelaide, Australia, 1997.

[8] H. Sequenz, Die Wicklungen elektrischer Maschinen, Springer-Verlag, Wien, 1950.

[9] B. Sobhi-Najafabadi Optimal Design of Permanent Magnet Generators, PhD Thesis, University of South Australia, Adelaide, Australia, 2002. 